\title{
Check-Operators and Quantum Spectral Curves
}

\author{
Andrei MIRONOV ${\dagger^{1} \dagger^{2} \dagger^{3} \dagger^{4}}^{4}$ and Alexei MOROZOV ${ }^{\dagger^{2} \dagger^{3} \dagger^{4}}$ \\ $\dagger^{1}$ Lebedev Physics Institute, Moscow, 119991, Russia \\ E-mail: mironov@lpi.ru \\ $\dagger^{2}$ ITEP, Moscow, 117218, Russia \\ E-mail: morozov@itep.ru \\ $\dagger^{3}$ Institute for Information Transmission Problems, Moscow, 127994, Russia \\ $\dagger^{4}$ National Research Nuclear University MEPhI, Moscow, 115409, Russia
}

Received January 29, 2017, in final form June 19, 2017; Published online June 26, 2017

https://doi.org/10.3842/SIGMA.2017.047

\begin{abstract}
We review the basic properties of effective actions of families of theories (i.e., the actions depending on additional non-perturbative moduli along with perturbative couplings), and their description in terms of operators (called check-operators), which act on the moduli space. It is this approach that led to constructing the (quantum) spectral curves and what is now nicknamed the EO/AMM topological recursion. We explain how the non-commutative algebra of check-operators is related to the modular kernels and how symplectic (special) geometry emerges from it in the classical (Seiberg-Witten) limit, where the quantum integrable structures turn into the well studied classical integrability. As time goes, these results turn applicable to more and more theories of physical importance, supporting the old idea that many universality classes of low-energy effective theories contain matrix model representatives.
\end{abstract}

Key words: matrix models; check-operators; Seiberg-Witten theory; modular kernel in CFT 2010 Mathematics Subject Classification: 14H70; 81R10; 81R12; 81T13

\section{Introduction}

One of the main lessons that we learned from string theory is that instead of working with a particular model (string or field), one should better consider families of similar models at once. This provides the most effective description of the problem, no matter has one to integrate over the space of theories (as in string theory) or not (as in the ordinary quantum field theory). This approach allows one to reveal various non-trivial structures underlying the family of theories that require involving a dynamics on the moduli space of theories. The most instructive examples of these structures are the algebras of constraints (Ward identities), that act on the partition functions of the theories $[10,25,31,42,61,76,91]$ and various integrable structures, from the standard integrability [33, 47, 48, 50, 64, 66, 67, 68, 69, 82, 83] to the Whitham integrability [49, 72].

Technically, one of the most effective tools turned out to be matrix models, working out these simple examples it was possible to develop a notion of check-operators. These operators act on the moduli space, and it turned out to be possible to mimic the action of various operators of the concrete theory by an action of check-operators [3, 4]. This framework allowed one later to realize many essential structures, from the topological recursion $[5,6,40,116]$ to dualities [44], from the wall-crossing formulas [45] to knot theory [45, 46, 51, 52, 53, 54, 55]. Moreover, it turns out that

This paper is a contribution to the Special Issue on Combinatorics of Moduli Spaces: Integrability, Cohomology, Quantisation, and Beyond. The full collection is available at http://www.emis.de/journals/SIGMA/modulispaces-2016.html 
this approach provides a simple description of some standard objects like modular kernels [44] and of some standard procedures like quantization of Seiberg-Witten integrabilities [81], both the standard $[89,92,110]$ and Whitham $[49,72,81]$ ones. As predicted $[84,85,87,103,104,105$, 106], nowadays they proved important far beyond the matrix model context, where they were originally discovered. The same will definitely happen to the new insights of general value from matrix model theory, which we mostly restrict to in the present review. While 25 years ago one mostly dealt with integrability, Virasoro constraints (or loop equations, or Ward identities) genus expansions and spectral curves, today the top issues are check-operators, their action on spaces of solutions to the loop equations, the quantum spectral curves and quantized Whitham flows.

Returning to applications, these include, first of all, topological strings. Here one considers matrix models of a quite general form which are referred to as matrix model networks [14, 70, 98, 99, 100, 107]. These matrix model networks have many avatars: on one hand, they may serve as a tool to study (refined) topological strings [1, 11, 12, 13, 37, 56, 57, 58, 59, 115] and Nekrasov functions [41, 108, 109], on another hand, within the framework of quantum field theory, they describe supersymmetric quiver gauge theories of Seiberg-Witten type, and, at last, at the algebraic level their partition functions are associated [30, 38, 39, 60, 90, 94, 95, 96] with the conformal blocks of Virasoro/W and Ding-Iohara-Miki algebras. Interrelations between these subject is nothing but the AGT correspondence [2, 30, 38, 39, 60, 90, 93, 94, 95, 96, 122].

One can illustrate these relations with the best studied example of two-parametric deformations of Seiberg-Witten (SW) systems:

\begin{tabular}{|c|c|c|c|}
\hline gauge theory & integrable system & spectral curve & AGT dual \\
\hline $\begin{array}{c}\text { Nekrasov function }\left(\epsilon_{1}, \epsilon_{2}\right) \\
\downarrow \epsilon_{2} \rightarrow 0\end{array}$ & $\begin{array}{c}\text { quantum integrable systems } \\
+ \text { Whitham flows } \\
\downarrow \epsilon_{1} \rightarrow 0\end{array}$ & $\begin{array}{c}\text { degenerate conformal } \\
\text { block equation }\end{array}$ & $\begin{array}{c}\text { conformal matrix } \\
\text { model, KP hierarchy }\end{array}$ \\
\hline $\begin{array}{c}\text { quantum Seiberg-Witten system } \\
\text { quantum many-body } \\
\text { integrable system, } \hbar=\epsilon_{1}\end{array}$ & $\begin{array}{c}\text { quantum spectral curve } \\
\text { Schrödinger (Baxter) } \\
\text { equation }\end{array}$ & $\begin{array}{c}\text { spectral dual } \\
\text { to quantum } \\
\text { integrable system }\end{array}$ \\
\hline Seiberg-Witten system & $\begin{array}{c}\text { classical finite-dimensional } \\
\text { integrable system }\end{array}$ & $\begin{array}{c}\text { spectral curve } \\
\text { (+ Whitham flows) }\end{array}$ & $\begin{array}{c}\text { spectral dual } \\
\text { to classical } \\
\text { integrable system }\end{array}$ \\
\hline
\end{tabular}

Below we consider only the simplest realization of check-operators and their applications within the ordinary matrix models, not the matrix model networks. We demonstrate how to construct check-operators, to obtain SW systems and to generate quantum spectral curves using them. We also describe a simple application of check-operators: a derivation of the modular kernel in two-dimensional conformal field theory.

This paper is a review of earlier results mostly described in [3, 4, 7, 8, 44]. In particular, the check-operators (see examples in Sections 3.1 and 3.4 below) were introduced in [3, 4], where their properties were discussed. Among the check-operators, there is the main check-operator (see Section 3.5) with the crucial property (3.1). In a different situation, this main check-operator was discussed in [44].

\section{Multiple solutions to the Virasoro constraints}

\subsection{Simplest example: the Hermitean matrix integral}

Thus, we start with the simplest example of the Hermitean matrix model

$$
Z=\int \mathrm{d} M \exp [\operatorname{Tr} V(M)],
$$

where we parameterize

$$
V(M)=\sum_{k=0} t_{k} M^{k}
$$


so that there is a natural grading $\left[t_{k}\right]=k$ and $\mathrm{d} M$ is the Haar measure of integration over $N \times N$ Hermitean matrices normalized to the volume of the unitary group $U(N) . t_{k}$ are here coefficients in the potential $V(M)$ which can be treated either as a formal series or as a polynomial of large enough degree (in the sense of the projective limit).

This integral satisfies an infinite set of Virasoro constraints (= loop equations) [10, 25, 31, $42,61,76,91]$ :

$$
\begin{aligned}
& L_{n} Z=0, \quad n \geq-1, \\
& L_{n}=\sum t_{k} \frac{\partial}{\partial t_{k+n}}+\sum_{a+b=n} \frac{\partial^{2}}{\partial t_{a} \partial t_{b}}, \\
& \frac{\partial Z}{\partial t_{0}}=N Z .
\end{aligned}
$$

These operators $L_{n}$ form a Borel subalgebra of the Virasoro algebra.

\subsection{Solutions as formal series}

The matrix model partition function is defined in (2.1) by a formal integral, which is still to be defined. Instead of this, we define it as any solution to the constraints (2.2). We also need to fix a class of functions where we look for these solutions. Let us consider power series solutions in all $t_{k}$, i.e., we treat the integral (2.1) perturbatively with respect to the potential $V(M)$. Then, there are no solutions to (2.2) at all! This is because the moments $\left\langle M^{k}\right\rangle$ all diverge. One has to regularize them.

The simplest way to do this is to consider the Gaussian integral, i.e., make the substitution $t_{2} \rightarrow t_{2}-\alpha$. Then,

$$
\begin{aligned}
Z & =\int \mathrm{d} M \exp \left[-\alpha \operatorname{Tr} M^{2}+\operatorname{Tr} V(M)\right]=c_{0}+c_{1} t_{1}+c_{2}^{(1)} t_{1}^{2}+c_{2}^{(2)} t_{2}+\cdots \\
& =\sum_{n} \sum_{\Delta:|\Delta|=n} c_{n}^{\Delta} t_{\Delta},
\end{aligned}
$$

where $c$ 's are some coefficients of grading $n$, which are constructed from the moments

$$
\int \mathrm{d} M \exp \left[-\alpha \operatorname{Tr} M^{2}\right] M^{k}
$$

and $\Delta$ is the Young diagram with lengths $\delta_{1} \geq \delta_{2} \geq \cdots \geq \delta_{k}, t_{\Delta}=\prod_{i=1}^{k} t_{\delta_{i}}$. Note that

$$
c_{i} \sim \alpha^{-i / 2}
$$

which is evident by dimensional argument.

Parameter $\alpha$ is the simplest example of a non-perturbative modulus. It is treated differently from the perturbative couplings $t_{k}$, but not independent of them. It can appear in denominators of the coefficients, still $\frac{\partial Z(\alpha \mid t)}{\partial \alpha}=-\frac{\partial Z(\alpha \mid t)}{\partial t_{2}}$.

\subsection{More general (Dijkgraaf-Vafa) case}

Let us now consider a more general Dijkgraaf-Vafa (DV) case, when a few first coefficients $t_{k}$ are shifted $t_{k} \longrightarrow T_{k}+t_{k}$ so that the partition function (2.1) becomes [27, 28, 29]

$$
Z_{\mathrm{DV}}=\int \mathrm{d} M \exp [\operatorname{Tr} W(M)+\operatorname{Tr} V(M)]
$$


where $W(M)=\sum^{p} T_{k} M^{k}$, and the integral is treated as a power series in $t_{k}$ 's, but as a function of $T_{k}$ 's. One also has to make the shift $t_{k} \longrightarrow T_{k}+t_{k}$ in the Virasoro constraints.

Then, the coefficients are constructed from

$$
\int \mathrm{d} M \exp [\operatorname{Tr} W(M)] M^{k}
$$

i.e., combinations of $T_{k}$ 's appear in denominators. One understands these integrals as integrals over properly chosen contours at fixed $W(M)$. This means that the matrices may not be literally Hermitean, since their eigenvalues are not obligatory real.

\subsection{How many solutions?}

Now one can enumerate the solutions to $(2.2)$ with $p$ first $t_{k}$ 's shifted $[7,8]$. One can state that solutions are parameterized by an arbitrary function of $p-2$ variables $T_{k}$. The two variables are fixed by the only linear constraints

$$
L_{0} Z=0, \quad L_{-1} Z=0,
$$

and all other constraints do not impose more restrictions, but serve as recurrence relations that allow one to evaluate all coefficients $c$. Thus, for $p=2$ (the Gaussian case) there is a unique solution.

\subsection{Technical tools: loop equations}

One can rewrite the constraints (2.2) by introducing a generating functions of the connected correlators (resolvents) in the model (2.3):

$$
\begin{aligned}
& \rho^{(1)}(z)=\left\langle\operatorname{Tr} \frac{1}{z-M}\right\rangle=\sum_{k=0}^{\infty} \frac{1}{z^{k+1}}\left\langle\operatorname{Tr} M^{k}\right\rangle=\frac{1}{Z_{\mathrm{DV}}} \hat{\nabla}_{z} Z_{\mathrm{DV}}=\hat{\nabla}_{z} \mathcal{F}, \\
& \rho^{(2)}\left(z_{1}, z_{2}\right)=\left\langle\operatorname{Tr} \frac{1}{z_{1}-M} \operatorname{Tr} \frac{1}{z_{2}-M}\right\rangle_{c}=\hat{\nabla}_{z_{1}} \hat{\nabla}_{z_{2}} \mathcal{F}, \\
& \ldots,
\end{aligned}
$$

where

$$
\hat{\nabla}_{z}=\sum_{k=0}^{\infty} \frac{1}{z^{k+1}} \frac{\partial}{\partial t_{k}}, \quad Z_{\mathrm{DV}}=\exp \mathcal{F}
$$

Then, the generating function for the constraints (2.2)

$$
\left[T(z) Z_{\mathrm{DV}}\right]_{-}=0, \quad T(z) \equiv \sum_{n=-\infty}^{+\infty} \frac{L_{n}}{z^{n+2}}
$$

can be rewritten in the form of loop equation

$$
\rho^{(1)}(z)^{2}+\hat{\nabla}_{z} \rho^{(1)}(z)+W^{\prime}(z) \rho^{(1)}(z)+\underbrace{\left[W^{\prime}(z) \rho^{(1)}(z)\right]_{+}}_{\text {polynomial of degree } p-2}+\underbrace{\left[V^{\prime}(z) \rho^{(1)}(z)\right]_{-}}_{=0 \text { as } t_{k} \rightarrow 0}=0 .
$$

Here the indices "+" and "-" denote the non-negative power and negative power parts of the expression accordingly. 


\section{Check-operators}

\subsection{Check-operator: acting on the space of solutions}

At all $t_{k}=0$, the last term in (2.4) vanishes, while the forth one

$$
f_{p-2}(z)=\left[W^{\prime}(z) \rho^{(1)}(z)\right]_{+}
$$

can be realized by the action of an operator $\check{R}_{z}$ in variables $T_{k}$ which are moduli of solutions

$$
f_{p-2}(z) \equiv \check{R}_{z} \mathcal{F}, \quad \check{R}_{z}=-\sum_{a, b}(a+b+2) T_{a+b+2} z^{a} \frac{\partial}{\partial T_{b}}
$$

This is the first example of a check-operator [7, 8].

This operator is crucially important to make the loop equations closed: $z$-dependence of $\rho$ depends on the action of $\check{R}_{z}$. At the same time, it affects the equation only "a little": the corresponding piece is a polynomial of finite degree in $z$, while the function $\rho^{(1)}(z)$ is essentially non-polynomial (in fact, one would better consider $\rho^{(1)}(z) d z$ as a 1-differential $[5,6,40,116]$, however, we do not discuss this kind of subtleties in the short review).

\subsection{Classical spectral curve}

Now let us define the classical spectral curve that describes the family of solutions to the matrix model $^{1}$. To this end, one has to make the genus expansion by rescaling the variables

$$
\left(t_{k}, T_{k}\right) \rightarrow\left(\frac{1}{g} t_{k}, \frac{1}{g} T_{k}\right), \quad Z=\exp \left(\frac{1}{g^{2}} \mathcal{F}\right)
$$

and considering the free energy expansion

$$
\mathcal{F}=\sum_{k} g^{2 k} \mathcal{F}_{k}
$$

The leading term (planar limit) of this genus expansion (which corresponds to neglecting the second term in (2.4)) in the resolvent is

$$
\rho_{0}^{(1)}(z)=\frac{-W^{\prime}(z)+y(z)}{2}
$$

at all $t_{k}=0$, where

$$
y(z)^{2} \equiv W^{\prime}(z)^{2}-4 f_{p-2}(z)
$$

determines the classical spectral curve. Generically, it is a hyperelliptic Riemann surface of genus $p-2$. Note that this resolvent is defined as the generating function of correlators that are not just power series but functions of $T_{k}$ 's.

Thus, the role of the check operator $\check{R}_{z}$ is exactly to provide the spectral curve. Loop equations then build $\rho(z)$ from this curve by a specially devised canonical procedure, known as the AMM/EO topological recursion [5, 6, 40, 116]. Remarkably, as we demonstrate below, these same check operators describe not only the classical spectral curve, but also its quantization.

\footnotetext{
${ }^{1}$ We remind here again that by the matrix model we mean the set of constraints $(2.2)$.
} 


\subsection{Examples}

Let us consider a couple of simplest examples.

- Gaussian case: $p=2, f_{0}(z)=$ const, $y^{2}=z^{2}$ - const. This leads to the notorious semi-circle distribution [36], and the Riemann surface has genus 0, it is just sphere.

- Cubic polynomial $W_{3}$ case: $p=3, f_{1}(z)$ is a linear function, the spectral curve is a torus, the space of solutions is described by a function of one variable.

Let us discuss a meaning of this last example [88]. In this case $p=3$, and, in accordance with general theory, the solutions to the Virasoro constraints (2.2) are fixed by a choice of an arbitrary function of one variable. What does this mean in terms of matrix integral?

For the eigenvalue matrix models it reduces to an $N$-fold integral over eigenvalues $x_{i}$ of $M$. Each of them, $\int \mathrm{d} x \mathrm{e}^{W_{3}(x)}$, depends on the choice of integration contours. For this cubic exponential, there are two independent contours (corresponding to two Airy functions), and any contour is an arbitrary linear combination of these two. Thus, the partition function is expanded into basic partition function with $N$ eigenvalues in the integrand parted into two groups (two possible contours) consisting of $N_{1}$ and $N_{2}$ eigenvalues, $N_{1}+N_{2}=N$. This describes the two-cut (torus) solution, and there is only one independent variable, say, the fraction $N_{1} / N_{2}$. This is why the solutions are parameterized by an arbitrary function of one variable. Increasing the degree of $W$ we get more and more independent integration contours and thus more and more moduli in the space of solutions.

\subsection{Summary of general properties}

Now we can formulate the general properties of solutions to the Virasoro constraints (2.2) at fixed $p$ and their moduli space $[3,4]$.

i) Any solution is unambiguously labeled by an arbitrary function of $p-2 T$-variables. This function can be associated with the free energy at all $t_{k}=0$. We call it the bare free energy $\mathcal{F}^{(0)}(T)$.

ii) Solutions to the Virasoro constraints (or loop equations) are constructed from $\mathcal{F}^{(0)}(T)$ by an evolution operator $\hat{U}(T, t)$ that does not depend on $\mathcal{F}^{(0)}(T)$ :

$$
Z(T, t)=\hat{U}(T, t) \mathrm{e}^{\mathcal{F}^{(0)}(T)}
$$

iii) The evolution operator $\hat{U}(T, t)$ is understood here as a power series in $t_{k}$ with the coefficients which can be completely expressed in terms of the unique operator $\check{R}(x)$ with its non-local "function" $\check{y}$

$$
\check{y} \equiv \sqrt{W^{\prime}(x)^{2}-4 \check{R}(x)}, \quad \check{R}(x) \equiv-\sum_{a, b=0}(a+b+2) T_{a+b+2} x^{a} \frac{\partial}{\partial T_{b}},
$$

its derivatives and $W^{\prime}(x)$. Here $\check{y}$ is defined as a power series at large $x$, see $[3,4]$ for the details.

\subsection{Main check-operator}

These general properties have an immediate consequence: they allow one to introduce the notion of the main check-operator $[3,4]$. Indeed, one can construct the resolvent from the free energy 
not only by the standard loop operator $\hat{\nabla}_{z}(t)$ acting on $t_{k}$ 's, but also by a check-operator acting on the moduli $T_{k}$ :

$$
\rho^{(1)}(z)=\hat{\nabla}_{z}(t) \mathcal{F}=\check{\nabla}_{z}(T) \mathcal{F}
$$

This check operator is called main, and it is for construction of this operator from the spectral curve "bundle" over the moduli space, that the AMM/EO recursion procedure was later devised $[5,6,40,116]$. It follows from the previous subsection that the main check-operator $\check{\nabla}_{z}$ is expressed through $y$, its derivatives and $W^{\prime}(x)$. It is important to notice that $\left[\hat{\nabla}_{z_{1}}, \hat{\nabla}_{z_{2}}\right]=0$, but $\left[\check{\nabla}_{z_{1}}, \check{\nabla}_{z_{2}}\right] \neq 0$. Hence, these operators are of different level of complexity, but the checkoperator acts on a much smaller space. Unfortunately, many properties of the check-operator have not been well-studied yet, though some of them are already known.

\subsection{Main property}

It turns out that the main check-operator possesses a very crucial property $[3,4]$ :

$$
\left[\oint_{A_{i}} \mathrm{~d} z \check{\nabla}_{z}, \oint_{B_{j}} \mathrm{~d} z \check{\nabla}_{z}\right]=\delta_{i j},
$$

where $A_{i}$ and $B_{i}$ are the $A$ - and $B$-cycles over the classical spectral curve $y^{2}=W^{\prime 2}(x)-$ $4 \check{R}(x) \mathcal{F}^{(0)}$ and the statement has been checked at the vicinity of large $x$, i.e., it requires a kind of analytic continuation to the whole spectral curve.

\section{Seiberg-Witten (SW) like solutions and integrable properties}

\subsection{DV/SW system}

The main check-operator property (3.1) immediately leads to the SW structure of the matrix models $[22,23,24,27,28,29]$. Indeed, choose the basis of functions parameterizing the space of solutions to the Virasoro constraints (2.2) to be eigenfunctions of the $A$-periods of the main check-operator: $\oint_{A_{i}} \mathrm{~d} z \check{\nabla}_{z} Z_{a}=a_{i} Z_{a}$, i.e., $\oint_{A_{i}} \mathrm{~d} z \check{\nabla}_{z} \mathcal{F}_{a}=\oint_{A_{i}} \mathrm{~d} z \rho^{(1)}(z)=a_{i}$, then

$$
\oint_{B_{i}} \rho^{(1)}(z) \mathrm{d} z=\frac{\partial \mathcal{F}_{a}}{\partial a_{i}}
$$

In the matrix model terms, the filling numbers $N_{i}$ 's that we discussed in Section 3.3 are associated with

$$
a_{i}=\oint_{A_{i}} \rho^{(1)}(z) \mathrm{d} z
$$

Let us stress again that we consider all the objects being formal series w.r.t. variables $t_{k}$ 's, but functions of $T_{k}$ 's, $N_{i}$ 's, $a_{i}$ 's.

\subsection{Integrable properties}

As usual, the matrix models have clear integrable properties:

- $Z(N \mid t)(2.1)$ is a $\tau$-function of the Toda chain (as a formal series) with $N$ playing role of the discrete time $[47,64,66,67,68,69]$, while, in the DV case, it is a sum of $Z_{a}$ (introduced in the previous subsection) which is this $\tau$-function [97]. 
- $Z_{\mathrm{DV}}\left(T_{k}, N_{i}\right)$ determines the SW system; hence, it satisfies the Whitham hierarchy [49, 72] in the planar limit, and $T_{k}$ are Whitham flows [22, 23, 24].

- In the planar limit, $Z_{\mathrm{DV}}\left(T_{k}, N_{i}\right)$ as a function of $T_{k}$ and $N_{i}$ also satisfies $[22,23]$ the WDVV equations [32, 35, 121], which is typical for $\tau$-functions of Whitham hierarchies, and for the SW systems [78, 79, 80, 86].

- The Dijkgraaf-Vafa partition function $Z_{\mathrm{DV}}\left(T_{k}, N_{i}\right)$ as the SW system is also associated with a many-body integrable system, classical [33, 48, 50, 82, 83] in the planar limit or quantum [81, 89, 92, 110], maybe even with the Whitham flows quantized [81] (see table in the Introduction). This quantization is realized by the check-operators.

\section{Quantum spectral curves}

The integrability of our matrix model allows one to define immediately the quantum spectral curve as an operator which cancels the Baker-Akhiezer function of the integrable system [47, 64, 66, 67, 68, 69, 71, 114]. Indeed, in our Toda chain case, the latter is defined through the $\tau$-function (the matrix model partition function) as (in this subsection $V$ denotes the potential with shifted coefficients, i.e., is a sum of $W+V$ in (2.3), and all the statements are treated in terms of formal series in $V$ )

$$
\Psi_{\mathrm{BA}}(z)=\mathrm{e}^{V(z) / 2} \Psi(z),
$$

where

$$
\Psi(z)=\frac{Z\left(t_{k}-\frac{1}{k z^{k}}\right)}{Z(t)}=\frac{1}{Z(t)} z^{N} \mathrm{e}^{\int^{z} \mathrm{~d} \xi \hat{\nabla}_{\xi}} Z(t)=\langle\operatorname{det}(z-M)\rangle .
$$

Here $\langle\cdots\rangle$ means the matrix model average. Since the Baker-Akhiezer function is proportional to the matrix model average of the determinant, one of the lessons is that this average also satisfies the quantum spectral curve equation.

From the Virasoro constraints (2.2), the quantum spectral curve looks like

$$
\left[\partial_{z}^{2}+V^{\prime}(z) \partial_{z}+\check{R}_{z}\right] \Psi(z)=0
$$

and, then, the equation for the Baker-Akhiezer function is

$$
\left[\partial_{z}^{2}-\frac{1}{2} V^{\prime \prime}(z)+\frac{1}{4} V^{\prime}(z)^{2}-\frac{1}{2}\left[\check{R}_{z} V(z)\right]+\check{R}_{z}\right] \Psi_{\mathrm{BA}}(z)=0 .
$$

In the classical (planar) limit, $\partial \log \Psi(z)=\rho_{0}^{(1)}(z)$ and equation (5.2) turns into the classical spectral curve (planar loop equation):

$$
\rho_{0}^{(1)}(z)^{2}+V^{\prime}(z) \rho_{0}^{(1)}(z)+\check{R}_{z} \mathcal{F}=0 .
$$

Note that, in integrable terms, $\check{R}_{z}$ contains the derivatives w.r.t. the Whitham times.

\section{Quantum curves from degenerate conformal blocks}

In the previous sections, we demonstrated what is the check-operator technique in the simplest example of matrix models. In the next two sections we illustrate it in a more involved example of two-dimensional conformal field theories [9, 21, 26, 102, 123]. The conformal block in this theory is also described by a matrix model, however, being a function, not just a formal series has more tricky global behaviour. This is one of the avatars of the AGT correspondence [2, 93, 122], which implies that the conformal block can be described as a $\beta$-ensemble of the Dotsenko-Fateev type [30, 34, 38, 39, 60, 90, 94, 95, 96]. 


\subsection{AGT and degenerate conformal blocks: quantum spectral curve}

Conformal block. The $n$-point conformal block $G\left(x_{k}, \Delta ; \Delta_{i}, c\right)[9,21,26,102,123]$ depends on the external conformal dimensions $\Delta_{i}$, on the internal dimension $\Delta$, on the central charge $c$ and on $n-3$ double ratios $x_{k}$ of points. These variables are most conveniently parameterized (in particular, from the point of view of the AGT correspondence) as $\Delta=(Q-\alpha) \alpha, c=1+6 Q^{2}$, $Q=b-1 / b$, the primary fields can be written in terms of the free field $\phi(z)$ as $V_{\alpha}(z)=: \mathrm{e}^{i \alpha \phi(z)}$ : and $: \ldots$ : denotes the normal ordering.

Degenerate conformal block and the spectral curve. Let us suppose that one of the fields in the conformal block is degenerate at a level $L$, which means it is simultaneously a primary field and a level $L$ descendant. Then, the corresponding conformal block satisfies an equation of order $L[9,21,26,102,123]$. For instance, $\left(b^{2} L_{-1}^{2}-L_{-2}\right) V_{1 / 2 b}(z)$ is a primary field, i.e., $V_{1 / 2 b}(z)$ is degenerate at the second level. Then, the equation for the 5-point block with the degenerate field at $z$ :

$$
[b^{2} z(z-1) \partial_{z}^{2}+(2 z-1) \partial_{z}-\underbrace{\frac{q(q-1)}{z-q} \partial_{q}+\text { rational function of } q}_{\text {check-operator }}] G_{5}(z \mid 0, q, 1, \infty)=0,
$$

where $q$ is the double ratio of four other points and we placed three points at 0,1 and $\infty$. This is the quantum spectral curve, while $q$ is a counterpart of $T_{k}$.

Comment on Toda quantum spectral curve. In the limit when all $\Delta_{i} \rightarrow \infty$, this equation is reduced to the non-stationary Schrödinger $\mathrm{SU}(2)$ periodic Toda chain equation

$$
\left(\partial_{z}^{2}-2 \Lambda^{2} \cosh z+\frac{1}{4} \frac{\partial}{\partial \Lambda}\right) G_{5}^{\text {Toda }}=0
$$

where $\Lambda$ is the limit of a properly rescaled variable $q$. This is the quantum spectral curve for the $\mathrm{SU}(2)$ periodic Toda chain, while $\log \Lambda$ is known to play the role of the first Whitham time in the Seiberg-Witten theory.

\subsection{Conformal matrix model}

Now let us note that the quantum spectral curve (6.1) is the curve for a matrix model, namely for the conformal matrix model $[15,16,17,30,38,39,60,65,77,90,94,95,96,101]$ :

$$
G_{4}(0, q, 1, \infty)=q^{2 \alpha_{1} \alpha_{2}}(1-q)^{2 \alpha_{2} \alpha_{3}} \int \prod_{i} \mathrm{~d} u_{i} \Delta^{2 b^{2}}(u) u_{i}^{2 b \alpha_{1}}\left(1-u_{i}\right)^{2 b \alpha_{3}}\left(q-u_{i}\right)^{2 b \alpha_{2}},
$$

where $\Delta(u)$ is the Van-der-Monde determinant and the integrals over $u_{i}$ 's part into groups: there are two integration contours, $[0, q]$ and $[0,1]$. Then, $\alpha, \alpha_{4}$ are related to the number of these contours:

- there are $N_{1}$ contours $[0, q]$ with

$$
b N_{1}=\alpha-\alpha_{1}-\alpha_{2}
$$

- there are $N_{2}$ contours $[0,1]$ with

$$
b N_{2}=Q-\alpha-\alpha_{3}-\alpha_{4} .
$$

$N_{1}$ and $N_{2}$ are associated with the Dijkgraaf-Vafa $N_{i}$ (see Section 3.3). Since the $\beta$-ensemble (6.2) can be presented in the form

$$
G_{4}=\left\langle V_{\alpha_{1}}(0) V_{\alpha_{2}}(q) V_{\alpha_{3}}(1) V_{\alpha_{4}}(\infty)\left(\int_{0}^{q} V_{b}(u) \mathrm{d} u\right)^{N_{1}}\left(\int_{0}^{1} V_{b}(u) \mathrm{d} u\right)^{N_{2}}\right\rangle_{\mathrm{CFT}},
$$


where $\langle\cdots\rangle_{\text {CFT }}$ denotes averaging in the free field theory and $\int V_{b}(u) \mathrm{d} u$ is a screening charge, it is nothing but the four-point conformal block [30, 38, 39, 60, 90, 94, 95, 96]. At the same time, the degenerate five-point conformal block $G_{5}=\left\langle V_{1 / 2 b}(z) \cdots\right\rangle_{\mathrm{CFT}}$. Since $\left\langle V_{1 / 2 b}(z) V_{b}(u)\right\rangle_{\mathrm{CFT}}=$ $z-u$, one immediately obtains $G_{5}=\left\langle\operatorname{det}\left(z-u_{i}\right)\right\rangle$. From (5.1) one knows that the matrix model average of the determinant satisfies the equation for the quantum spectral curve. Hence, the equation (6.1) for $G_{5}$ is exactly the quantum spectral curve for the Dotsenko-Fateev (or conformal) matrix model (6.2).

\section{Modular kernels in conformal field theory}

Now we are ready to use the developed technique to derive the modular kernel in conformal theory.

\subsection{Modular kernel for 4-point conformal block}

The modular kernel is defined for the modular transformation $S: x \rightarrow 1-x$ by the formula

$$
G_{4}\left(x, a ; a_{i}, b\right)=\int \mathrm{d} a^{\prime} K\left(a, a^{\prime} ; a_{i}, b\right) G_{4}\left(1-x, a^{\prime} ; a_{i}, b\right),
$$

and we use the notation $a_{i}=\alpha_{i}-Q / 2$.

Explicit expression for modular kernel. The explicit expression for the four-point conformal block was obtained by a tedious work in $[117,118]$ and has the form

$$
K\left(a, a^{\prime} ; a_{i}, b\right)=4 \sinh \left(2 \pi a^{\prime} / b\right) \sinh \left(2 \pi b a^{\prime}\right) \frac{S_{b}\left(u_{1}\right) S_{b}\left(u_{2}\right)}{S_{b}\left(v_{1}\right) S_{b}\left(v_{2}\right)} \int \mathrm{d} x \prod_{i=1}^{4} \frac{S_{b}\left(x-\xi_{i}\right)}{S_{b}\left(x-\zeta_{i}\right)},
$$

where $S_{b}(x)$ is the double sine function $[62,63,73,74,75,119], u_{i}, v_{i}, \xi_{i}, \zeta_{i}$ are linear functions of $a_{i}, b, a$ and $a^{\prime}$, and the choice of integration contours is quite tricky [117, 118].

Representation of $\boldsymbol{G}\left(\boldsymbol{x}, \boldsymbol{a} ; \boldsymbol{a}_{\boldsymbol{i}}, \boldsymbol{b}\right)$ as a $\boldsymbol{\beta}$-ensemble with $\boldsymbol{\beta}=\boldsymbol{b}^{\mathbf{2}}$. One can also calculate the modular kernel from the matrix model representation of the conformal block (6.2) perturbatively in the genus expansion term by term [43, 111], the result being quite surprising: the modular kernel in all orders of the expansion is the Fourier kernel:

$$
K\left(a, a^{\prime} ; a_{i}, b\right)=\mathrm{e}^{2 \pi i a a^{\prime}} .
$$

This results seems to contradict to the result of $[117,118]$, and we now explain the reason for the difference and derive the result of $[117,118]$ in a simple way in a simpler case of the one-point conformal block on torus.

\subsection{1-point toric conformal block}

We consider the one-point toric conformal block, which has the following series expansion

$$
G(\tau, a ; \mu)=1+q\left(\frac{\Delta_{\mathrm{ext}}\left(1-\Delta_{\mathrm{ext}}\right)}{2 \Delta}+1\right)+O\left(q^{2}\right)
$$

with $\Delta_{\text {ext }}=\mu(Q-\mu)$, and $q=\exp i \pi \tau, \tau$ being the torus modular parameter. In terms of the AGT dual gauge theory, $\mu$ is the adjoint hypermultiplet mass. The modular transformation of the conformal block now is given by the modular transformation of torus:

$$
G(\tau, a ; \mu)=\int \mathrm{d} a^{\prime} K\left(a, a^{\prime} ; \mu\right) G\left(-\tau^{-1}, a^{\prime} ; \mu\right) .
$$


Explicit expression for modular kernel. This time the explicit expression for the modular kernel due to [120] is

$$
K\left(a, a^{\prime} ; \mu\right) \sim \int \mathrm{d} \xi \frac{S_{b}\left(\xi+\mu / 2-a^{\prime}\right) S_{b}\left(\xi+\mu / 2+a^{\prime}\right)}{S_{b}\left(\xi+Q-\mu / 2-a^{\prime}\right) S_{b}\left(\xi+Q-\mu / 2+a^{\prime}\right)} \mathrm{e}^{4 \pi i a \xi} .
$$

Modular kernel from $\boldsymbol{\beta}$-ensemble. One can again get the modular kernel from the $\beta$ ensemble realization of the conformal block $[43,111]$. In this case, the essential point is that the conformal block differs from the partition function of the $\beta$-ensemble by a normalization factor [44]

$$
G(\tau, a ; \mu)=\frac{1}{N(a)} Z(\tau, a ; \mu), \quad N(a)=\frac{\Gamma_{b}(2 a+\mu) \Gamma_{b}(2 a+Q-\mu)}{\Gamma_{b}(2 a), \Gamma_{b}(2 a+Q)}
$$

where $\Gamma_{b}(x)$ is the Barnes double gamma function [18, 19, 20, 62, 63]. The partition function turns out again to be transformed in the genus expansion by the pure Fourier transform

$$
Z(\tau, a ; \mu)=\int \mathrm{d} a^{\prime} \mathrm{e}^{2 \pi i a a^{\prime}} Z\left(-\tau^{-1}, a^{\prime} ; \mu\right)
$$

i.e., the modular kernel appeared to be purely exponential. We now see why this is not quite the case and explain how to correct the calculation.

\subsection{An archetypical example}

An archetypical example of duality is provided by the pair of operators constructed from the coordinate and momentum, $\hat{A}=\mathrm{e}^{i \hat{\mathcal{P}}}$ and $\hat{B}=\mathrm{e}^{i \hat{\mathcal{Q}}}$, with the commutation relation

$$
\hat{A} \hat{B}=\mathrm{e}^{i \hbar} \hat{B} \hat{A}
$$

Then, their eigenfunctions are related by the Fourier transform in the eigenvalue space:

$$
\hat{A} Z_{a}(\mathcal{Q})=\mathrm{e}^{i a} Z_{a}(\mathcal{Q}), \quad \hat{B} \tilde{Z}_{a^{\prime}}(\mathcal{Q})=\mathrm{e}^{i a^{\prime}} \tilde{Z}_{a^{\prime}}(\mathcal{Q}) \stackrel{(7.2)}{\Longrightarrow} Z_{a}(\mathcal{Q})=\int \mathrm{e}^{\frac{i a a^{\prime}}{\hbar}} \tilde{Z}_{a^{\prime}}(\mathcal{Q}) \mathrm{d} a^{\prime},
$$

which can be easily checked by the direct calculation of the eigenfunctions:

$$
Z_{a}(\mathcal{Q})=\mathrm{e}^{\frac{i a \mathcal{Q}}{\hbar}}, \quad \tilde{Z}_{a^{\prime}}(\mathcal{Q})=\delta\left(\mathcal{Q}-a^{\prime}\right)
$$

Check-operators. One, however, does not need to calculate the eigenfunctions in order to determine what is the transformation kernel. Instead, one can substitute the two operators by their representatives in the eigenvalue space, which reproduce the right commutation relations:

$$
\check{A}=\mathrm{e}^{i a}, \quad \check{B}=\mathrm{e}^{\hbar \frac{\partial}{\partial a}} .
$$

Then the transformation kernel $M\left(a, a^{\prime}\right)=\mathrm{e}^{\frac{i a a^{\prime}}{\hbar}}$ is simply obtained from the equation

$$
\check{A}(a) M\left(a, a^{\prime}\right)=\check{B}\left(a^{\prime}\right) M\left(a, a^{\prime}\right) .
$$

\subsection{Conformal block as an eigenfunction}

The conformal block turns out to be an eigenfunction of some operator $\mathcal{L}_{A}$ :

$$
\mathcal{L}_{A} G=\lambda G, \quad \mathcal{L}_{B} G=\Lambda\left(\partial_{\lambda}\right) G,
$$


which is constructed, similarly to the previous subsection, from the canonical pair of operators [44]. Taking into account the matrix model ( $\beta$-ensemble) representation of the conformal block, it is natural that this pair is given by periods of the main check-operator, (3.1). Hence,

Claim.

$$
\mathcal{L}_{\gamma}=\mathrm{e}^{b \oint_{\gamma} \mathrm{d} z \check{\nabla}_{z}}
$$

Since $\left[\mathcal{L}_{A}, \mathcal{L}_{B}\right]=1$, one obtains that $K\left(a, a^{\prime} ; \mu\right)$ is the Fourier exponential. This is what was obtained perturbatively $[43,111]$, and it was a pretty tedious calculation!

Subtlety. Now one has to ask why (7.1) is not the exponential. The answer is hidden in the analytic properties of the partition function: the conformal theory is invariant with respect to the reflection $a \rightarrow-a$, but there are two different main check-operators

$$
\oint_{A} \mathrm{~d} z \check{\nabla}_{z}^{(+)} Z_{a}^{(+)}=a Z_{a}^{(+)}, \quad \oint_{A} \mathrm{~d} z \check{\nabla}_{z}^{(-)} Z_{a}^{(-)}=-a Z_{a}^{(-)},
$$

and two different branches of the $\beta$-ensemble partition function, i.e., $G$ is globally defined but $Z(a)$ is not! There are two branches at $a>0$ and $a<0$. Thus, one should naturally act with a sum of two exponentials of the two main check-operators and take into account the normalization factor $N(a)$ that recalculate the action of $\check{\nabla}_{z}$ from the partition function to the conformal block:

$$
\mathcal{L}_{\gamma}=\left[\frac{1}{N(a)} \mathrm{e}^{b \oint_{\gamma} \mathrm{d} z \check{\nabla}_{z}^{+}} N(a)+\frac{1}{N(-a)} \mathrm{e}^{-b \oint_{\gamma} \mathrm{d} z \check{\nabla}_{z}^{-}} N(-a)\right] .
$$

\subsection{Modular kernel for the torus conformal block}

Now we are ready to calculate the exact modular kernel [44]. First of all, one can realize the periods of check-operators in the space of eigenvalues similarly to (7.3):

$$
\oint_{A} \mathrm{~d} z \check{\nabla}_{z}^{ \pm} \rightarrow \pm 2 \pi i a, \quad \oint_{B} \mathrm{~d} z \check{\nabla}_{z}^{ \pm} \rightarrow \pm \frac{1}{2} \partial_{a}
$$

Thus, one obtains

$$
\mathcal{L}_{B}=\frac{\Gamma(2 a b) \Gamma(b Q+2 a b)}{\Gamma(b \mu+2 a b) \Gamma(b(Q-\mu)+2 a b)} \mathrm{e}^{\frac{b}{2} \partial_{a}}+(a \rightarrow-a) .
$$

Since $\mathcal{L}_{A}^{\prime}=\cos 2 \pi b a$, we can find the modular kernel from the equations (7.3), which becomes

$$
\frac{1}{2}\left(\frac{\sin 2 \pi b(a-\mu / 2)}{\sin 2 \pi b a} \mathrm{e}^{-\frac{b}{2} \partial_{a}}+\frac{\sin 2 \pi b(a+\mu / 2)}{\sin 2 \pi b a} \mathrm{e}^{\frac{b}{2} \partial_{a}}\right) K\left(a, a^{\prime}\right)=\cos 2 \pi b a^{\prime} K\left(a, a^{\prime}\right) .
$$

At large $a$, only one exponential survives giving the pure exponential kernel (see next corrections in $[113,112])$. The solution of the full equation is immediately constructed [44] and is given by

$$
K\left(a, a^{\prime} ; \mu\right)=\int \mathrm{d} \xi C_{1}(\xi) C_{2}\left(a^{\prime}\right) \frac{S_{b}\left(\xi+\mu / 2-a^{\prime}\right) S_{b}\left(\xi+\mu / 2+a^{\prime}\right)}{S_{b}\left(\xi+Q-\mu / 2-a^{\prime}\right) S_{b}\left(\xi+Q-\mu / 2+a^{\prime}\right)} \mathrm{e}^{4 \pi i a \xi},
$$

where $C_{1}(\xi)$ is an arbitrary periodic function with period $b$ and $C_{2}\left(a^{\prime}\right)$ is an arbitrary function. This result coincides with formula (4.41) in [120] at $C_{1}=C_{2}=1$. Further details can be found in $[112,113]$. 


\section{Conclusion}

In this review, we introduced and explained the very important notion of check-operator: the operator that acts on the moduli space of theories (or vacua/solutions). We constructed the operator manifestly in the simplest example of the Hermitian matrix model and in a more involved example of the two-dimensional conformal field theory, and demonstrated its use by deriving the corresponding Seiberg-Witten structures and the quantum spectral curves. We also illustrated the usefulness of the concept by a simple evaluation of the kernel of modular transformation of the conformal blocks done in terms of the check-operators. The calculation used the wonderful relation (3.1), which provides the impressive example of the properties and the relevance of check-operators for the quantization theory.

\section{Acknowledgements}

This work was performed at the Institute for Information Transmission Problems with the financial support of the Russian Science Foundation (Grant No.14-50-00150).

\section{References}

[1] Aganagic M., Klemm A., Mariño M., Vafa C., The topological vertex, Comm. Math. Phys. 254 (2005), 425-478, hep-th/0305132.

[2] Alday L.F., Gaiotto D., Tachikawa Y., Liouville correlation functions from four-dimensional gauge theories, Lett. Math. Phys. 91 (2010), 167-197, arXiv:0906.3219.

[3] Alexandrov A., Mironov A., Morozov A., Solving Virasoro constraints in matrix models, Fortschr. Phys. 53 (2005), 512-521, hep-th/0412205.

[4] Alexandrov A., Mironov A., Morozov A., Unified description of correlators in non-Gaussian phases of Hermitian matrix model, Internat. J. Modern Phys. A 21 (2006), 2481-2517, hep-th/0412099.

[5] Alexandrov A., Mironov A., Morozov A., Instantons and merons in matrix models, Phys. D 235 (2007), 126-167, hep-th/0608228.

[6] Alexandrov A., Mironov A., Morozov A., BGWM as second constituent of complex matrix model, J. High Energy Phys. 2009 (2009), no. 12, 053, 49 pages, arXiv:0906.3305.

[7] Alexandrov A., Mironov A., Morozov A., Putrov P., Partition functions of matrix models as the first special functions of string theory. II. Kontsevich model, Internat. J. Modern Phys. A 24 (2009), 4939-4998, arXiv:0811.2825.

[8] Alexandrov A., Morozov A., Mironov A., Partition functions of matrix models: first special functions of string theory, Internat. J. Modern Phys. A 19 (2004), 4127-4163, hep-th/0310113.

[9] Alvarez-Gaumé L., Random surfaces, statistical mechanics and string theory, Helv. Phys. Acta 64 (1991), $359-526$.

[10] Ambjørn J., Makeenko Yu.M., Properties of loop equations for the Hermitian matrix model and for twodimensional quantum gravity, Modern Phys. Lett. A 5 (1990), 1753-1763.

[11] Awata H., Kanno H., Instanton counting, Macdonald function and the moduli space of D-branes, J. High Energy Phys. 2005 (2005), no. 5, 039, 26 pages, hep-th/0502061.

[12] Awata H., Kanno H., Refined BPS state counting from Nekrasov's formula and Macdonald functions, Internat. J. Modern Phys. A 24 (2009), 2253-2306, arXiv:0805.0191.

[13] Awata H., Kanno H., Changing the preferred direction of the refined topological vertex, J. Geom. Phys. 64 (2013), 91-110, arXiv:0903.5383.

[14] Awata H., Kanno H., Matsumoto T., Mironov A., Morozov A., Morozov A., Ohkubo Y., Zenkevich Y., Explicit examples of DIM constraints for network matrix models, J. High Energy Phys. 2016 (2016), no. 7, 103, 67 pages, arXiv:1604.08366.

[15] Awata H., Matsuo Y., Odake S., Shiraishi J., Collective field theory, Calogero-Sutherland model and generalized matrix models, Phys. Lett. B 347 (1995), 49-55, hep-th/9411053. 
[16] Awata H., Matsuo Y., Odake S., Shiraishi J., Excited states of the Calogero-Sutherland model and singular vectors of the $W_{N}$ algebra, Nuclear Phys. B 449 (1995), 347-374, hep-th/9503043.

[17] Awata H., Matsuo Y., Odake S., Shiraishi J., A Note on Calogero-Sutherland model, $W_{n}$ singular vectors and generalized matrix models, Soryushiron Kenkyu 91 (1995), A69-A75, hep-th/9503028.

[18] Barnes E.W., The genesis of the double gamma functions, Proc. London Math. Soc. S1-31 (1899), 358-381.

[19] Barnes E.W., The theory of the double gamma function, Philos. Trans. R. Soc. Lond. Ser. A 96 (1901), $265-387$.

[20] Barnes E.W., On the theory of multiple gamma functions, Trans. Cambridge Philos. Soc. 19 (1904), 374-425.

[21] Belavin A.A., Polyakov A.M., Zamolodchikov A.B., Infinite conformal symmetry in two-dimensional quantum field theory, Nuclear Phys. B 241 (1984), 333-380.

[22] Chekhov L., Marshakov A., Mironov A., Vasiliev D., DV and WDVV, Phys. Lett. B 562 (2003), 323-338, hep-th/0301071.

[23] Chekhov L., Marshakov A., Mironov A., Vasiliev D., Complex geometry of matrix models, Proc. Steklov Inst. Math. 251 (2005), 265-306, hep-th/0506075.

[24] Chekhov L., Mironov A., Matrix models vs. Seiberg-Witten/Whitham theories, Phys. Lett. B 552 (2003), 293-302, hep-th/0209085.

[25] David F., Loop equations and nonperturbative effects in two-dimensional quantum gravity, Modern Phys. Lett. A 5 (1990), 1019-1029.

[26] Di Francesco P., Mathieu P., Sénéchal D., Conformal field theory, Graduate Texts in Contemporary Physics, Springer-Verlag, New York, 1997.

[27] Dijkgraaf R., Vafa C., Matrix models, topological strings, and supersymmetric gauge theories, Nuclear Phys. B 644 (2002), 3-20, hep-th/0206255.

[28] Dijkgraaf R., Vafa C., On geometry and matrix models, Nuclear Phys. B 644 (2002), 21-39, hep-th/0207106.

[29] Dijkgraaf R., Vafa C., A perturbative window into non-perturbative physics, hep-th/0208048.

[30] Dijkgraaf R., Vafa C., Toda theories, matrix models, topological strings, and $N=2$ gauge systems, arXiv:0909.2453.

[31] Dijkgraaf R., Verlinde H., Verlinde E., Loop equations and Virasoro constraints in nonperturbative twodimensional quantum gravity, Nuclear Phys. B 348 (1991), 435-456.

[32] Dijkgraaf R., Verlinde H., Verlinde E., Topological strings in $d<1$, Nuclear Phys. B 352 (1991), 59-86.

[33] Donagi R., Witten E., Supersymmetric Yang-Mills theory and integrable systems, Nuclear Phys. B 460 (1996), 299-334, hep-th/9510101.

[34] Dotsenko V.S., Fateev V.A., Conformal algebra and multipoint correlation functions in 2D statistical models, Nuclear Phys. B 240 (1984), 312-348.

[35] Dubrovin B., Geometry of 2D topological field theories, in Integrable Systems and Quantum Groups (Montecatini Terme, 1993), Lecture Notes in Math., Vol. 1620, Springer, Berlin, 1996, 120-348, hep-th/9407018.

[36] Dyson F.J., Statistical theory of the energy levels of complex systems. I, J. Math. Phys. 3 (1962), 140-156.

[37] Eguchi T., Kanno H., Topological strings and Nekrasov's formulas, J. High Energy Phys. 2003 (2003), no. 12, 006, 30 pages, hep-th/0310235.

[38] Eguchi T., Maruyoshi K., Penner type matrix model and Seiberg-Witten theory, J. High Energy Phys. 2010 (2010), no. 2, 022, 21 pages, arXiv:0911.4797.

[39] Eguchi T., Maruyoshi K., Seiberg-Witten theory, matrix model and AGT relation, J. High Energy Phys. 2010 (2010), no. 7, 081, 18 pages, arXiv:1006.0828.

[40] Eynard B., Orantin N., Invariants of algebraic curves and topological expansion, Commun. Number Theory Phys. 1 (2007), 347-452, math-ph/0702045.

[41] Flume R., Poghossian R., An algorithm for the microscopic evaluation of the coefficients of the SeibergWitten prepotential, Internat. J. Modern Phys. A 18 (2003), 2541-2563, hep-th/0208176.

[42] Fukuma M., Kawai H., Nakayama R., Continuum Schwinger-Dyson equations and universal structures in two-dimensional quantum gravity, Internat. J. Modern Phys. A 6 (1991), 1385-1406.

[43] Galakhov D., Mironov A., Morozov A., S-duality as a $\beta$-deformed Fourier transform, J. High Energy Phys. 2012 (2012), no. 8, 067, 28 pages, arXiv:1205.4998. 
[44] Galakhov D., Mironov A., Morozov A., $S$-duality and modular transformation as a non-perturbative deformation of the ordinary pq-duality, J. High Energy Phys. 2014 (2014), no. 6, 050, 24 pages, arXiv:1311.7069.

[45] Galakhov D., Mironov A., Morozov A., Wall crossing invariants: from quantum mechanics to knots, J. Exp. Theor. Phys. 120 (2015), 549-577, arXiv:1410.8482.

[46] Galakhov D., Mironov A., Morozov A., SU(2)/SL(2) knot invariants and Kontsevich-Soibelman monodromies, Theoret. and Math. Phys. 187 (2016), 678-694, arXiv:1510.05366.

[47] Gerasimov A., Marshakov A., Mironov A., Morozov A., Orlov A., Matrix models of two-dimensional gravity and Toda theory, Nuclear Phys. B 357 (1991), 565-618.

[48] Gorsky A., Krichever I.M., Marshakov A., Mironov A., Morozov A., Integrability and Seiberg-Witten exact solution, Phys. Lett. B 355 (1995), 466-474, hep-th/9505035.

[49] Gorsky A., Marshakov A., Mironov A., Morozov A., RG equations from Whitham hierarchy, Nuclear Phys. B 527 (1998), 690-716, hep-th/9802004.

[50] Gorsky A., Mironov A., Integrable many-body systems and gauge theories, hep-th/0011197.

[51] Hikami K., Hyperbolic structure arising from a knot invariant, Internat. J. Modern Phys. A 16 (2001), 3309-3333, math-ph/0105039.

[52] Hikami K., Generalized volume conjecture and the $A$-polynomials: the Neumann-Zagier potential function as a classical limit of the partition function, J. Geom. Phys. 57 (2007), 1895-1940, math.QA/0604094.

[53] Hikami K., Inoue R., Braiding operator via quantum cluster algebra, J. Phys. A: Math. Theor. 47 (2014), 474006, 21 pages, arXiv:1404.2009.

[54] Hikami K., Inoue R., Cluster algebra and complex volume of once-punctured torus bundles and 2-bridge links, J. Knot Theory Ramifications 23 (2014), 1450006, 33 pages, arXiv:1212.6042.

[55] Hikami K., Inoue R., Braids, complex volume and cluster algebras, Algebr. Geom. Topol. 15 (2015), 21752194, arXiv:1304.4776.

[56] Iqbal A., All genus topological string amplitudes and 5-brane webs as Feynman diagrams, hep-th/0207114.

[57] Iqbal A., Kashani-Poor A.K., The vertex on a strip, Adv. Theor. Math. Phys. 10 (2006), 317-343, hep-th/0410174.

[58] Iqbal A., Kozçaz C., Vafa C., The refined topological vertex, J. High Energy Phys. 2009 (2009), no. 10, 069, 58 pages, hep-th/0701156.

[59] Iqbal A., Vafa C., Nekrasov N., Okounkov A., Quantum foam and topological strings, J. High Energy Phys. 2008 (2008), no. 4, 011, 47 pages, hep-th/0312022.

[60] Itoyama H., Maruyoshi K., Oota T., Notes on the quiver matrix model and 2d-4d conformal connection, Progr. Theoret. Phys. 123 (2010), 957-987, arXiv:0911.4244.

[61] Itoyama H., Matsuo Y., Noncritical Virasoro algebra of the $d<1$ matrix model and the quantized string field, Phys. Lett. B 255 (1991), 202-208.

[62] Jimbo M., Miwa T., Quantum KZ equation with $|q|=1$ and correlation functions of the $X X Z$ model in the gapless regime, J. Phys. A: Math. Gen. 29 (1996), 2923-2958, hep-th/9601135.

[63] Kharchev S., Lebedev D., Semenov-Tian-Shansky M., Unitary representations of $U_{q}(\mathfrak{s l}(2, \mathbb{R}))$, the modular double and the multiparticle $q$-deformed Toda chains, Comm. Math. Phys. 225 (2002), 573-609, hepth/0102180.

[64] Kharchev S., Marshakov A., Mironov A., Morozov A., Generalized Kontsevich model versus Toda hierarchy and discrete matrix models, Nuclear Phys. B 397 (1993), 339-378, hep-th/9203043.

[65] Kharchev S., Marshakov A., Mironov A., Morozov A., Pakuliak S., Conformal matrix models as an alternative to conventional multi-matrix models, Nuclear Phys. B 404 (1993), 717-750, hep-th/9208044.

[66] Kharchev S., Marshakov A., Mironov A., Morozov A., Zabrodin A., Towards unified theory of 2d gravity, Nuclear Phys. B 380 (1992), 181-240, hep-th/9201013.

[67] Kharchev S., Marshakov A., Mironov A., Morozov A., Zabrodin A., Unification of all string models with $c<1$, Phys. Lett. B 275 (1992), 311-314, hep-th/9111037.

[68] Kharchev S., Marshakov A., Mironov A., Orlov A., Zabrodin A., Matrix models among integrable theories: forced hierarchies and operator formalism, Nuclear Phys. B 366 (1991), 569-601.

[69] Kharchev S., Mironov A., Integrable structures of unitary matrix models, Internat. J. Modern Phys. A 7 (1992), 4803-4824.

[70] Kimura T., Pestun V., Quiver W-algebras, arXiv:1512.08533. 
[71] Krichever I.M., Methods of algebraic geometry in the theory of non-linear equations, Russian Math. Surveys 32 (1977), no. 6, 185-213.

[72] Krichever I.M., The $\tau$-function of the universal Whitham hierarchy, matrix models and topological field theories, Comm. Pure Appl. Math. 47 (1994), 437-475, hep-th/9205110.

[73] Kurokawa N., Multiple sine functions and Selberg zeta functions, Proc. Japan Acad. Ser. A Math. Sci. 67 (1991), 61-64.

[74] Kurokawa N., Gamma factors and Plancherel measures, Proc. Japan Acad. Ser. A Math. Sci. 68 (1992), 256-260.

[75] Kurokawa N., Multiple zeta functions: an example, in Zeta Functions in Geometry (Tokyo, 1990), Adv. Stud. Pure Math., Vol. 21, Kinokuniya, Tokyo, 1992, 219-226.

[76] Makeenko Yu., Marshakov A., Mironov A., Morozov A., Continuum versus discrete Virasoro in one-matrix models, Nuclear Phys. B 356 (1991), 574-628.

[77] Marshakov A., Mironov A., Morozov A., Generalized matrix models as conformal field theories. Discrete case, Phys. Lett. B 265 (1991), 99-107.

[78] Marshakov A., Mironov A., Morozov A., WDVV-like equations in $\mathcal{N}=2$ SUSY Yang-Mills theory, Phys. Lett. B 389 (1996), 43-52, hep-th/9607109.

[79] Marshakov A., Mironov A., Morozov A., WDVV equations from algebra of forms, Modern Phys. Lett. A 12 (1997), 773-787, hep-th/9701014.

[80] Marshakov A., Mironov A., Morozov A., More evidence for the WDVV equations in $\mathcal{N}=2$ SUSY Yang-Mills theories, Internat. J. Modern Phys. A 15 (2000), 1157-1206, hep-th/9701123.

[81] Marshakov A., Mironov A., Morosov A., On AGT relations with surface operator insertion and a stationary limit of beta-ensembles, J. Geom. Phys. 61 (2011), 1203-1222, arXiv:1011.4491.

[82] Martinec E.J., Integrable structures in supersymmetric gauge and string theory, Phys. Lett. B 367 (1996), 91-96, hep-th/9510204.

[83] Martinec E.J., Warner N.P., Integrable systems and supersymmetric gauge theory, Nuclear Phys. B 459 (1996), 97-112, hep-th/9511052.

[84] Mironov A., 2D gravity and matrix models. I. 2D gravity, Internat. J. Modern Phys. A 9 (1994), 4355-4405, hep-th/9312212.

[85] Mironov A., Quantum deformations of $\tau$-functions, bilinear identities and representation theory, hep-th/9409190.

[86] Mironov A., WDVV equations and Seiberg-Witten theory, in Integrability: the Seiberg-Witten and Whitham Equations (Edinburgh, 1998), Editors H.W. Braden, I.M. Krichever, Gordon and Breach, Amsterdam, 2000, 103-123, hep-th/9903088.

[87] Mironov A., Matrix models of two-dimensional gravity, Phys. Part. Nuclei 33 (2002), 1051-1145.

[88] Mironov A., Matrix models and matrix integrals, Theoret. and Math. Phys. 146 (2006), 63-72, hepth/0506158.

[89] Mironov A., Morosov A., Nekrasov functions and exact Bohr-Sommerfeld integrals, J. High Energy Phys. 2010 (2010), no. 4, 040, 15 pages, arXiv:0910.5670.

[90] Mironov A., Morosov A., Shakirov S., Brezin-Gross-Witten model as "pure gauge" limit of Selberg integrals, J. High Energy Phys. 2011 (2011), no. 3, 102, 25 pages, arXiv:1011.3481.

[91] Mironov A., Morozov A., On the origin of Virasoro constraints in matrix models: Lagrangian approach, Phys. Lett. B 252 (1990), 47-52.

[92] Mironov A., Morozov A., Nekrasov functions from exact Bohr-Sommerfeld periods: the case of SU( $N)$, J. Phys. A: Math. Theor. 43 (2010), 195401, 11 pages, arXiv:0911.2396.

[93] Mironov A., Morozov A., On AGT relation in the case of U(3), Nuclear Phys. B 825 (2010), 1-37, arXiv:0908.2569.

[94] Mironov A., Morozov A., Morozov A., Conformal blocks and generalized Selberg integrals, Nuclear Phys. B 843 (2011), 534-557, arXiv:1003.5752.

[95] Mironov A., Morozov A., Shakirov S., Conformal blocks as Dotsenko-Fateev integral discriminants, Internat. J. Modern Phys. A 25 (2010), 3173-3207, arXiv:1001.0563.

[96] Mironov A., Morozov A., Shakirov S., Matrix model conjecture for exact BS periods and Nekrasov functions, J. High Energy Phys. 2010 (2010), no. 2, 030, 26 pages, arXiv:0911.5721. 
[97] Mironov A., Morozov A., Zakirova Z., Comment on integrability in Dijkgraaf-Vafa $\beta$-ensembles, Phys. Lett. B 711 (2012), 332-335, arXiv:1202.6029.

[98] Mironov A., Morozov A., Zenkevich Y., Ding-Iohara-Miki symmetry of network matrix models, Phys. Lett. B $\mathbf{7 6 2}$ (2016), 196-208, arXiv:1603.05467.

[99] Mironov A., Morozov A., Zenkevich Y., On elementary proof of AGT duality from six dimensions, Phys. Lett. B 756 (2016), 208-211, arXiv:1512.06701.

[100] Mironov A., Morozov A., Zenkevich Y., Spectral duality in elliptic systems, six-dimensional gauge theories and topological strings, J. High Energy Phys. 2016 (2016), no. 5, 121, 44 pages, arXiv:1603.00304.

[101] Mironov A., Pakuliak S., On the continuum limit of the conformal matrix models, Theoret. and Math. Phys. 95 (1993), 604-625, hep-th/9209100.

[102] Moore G., Seiberg N., Classical and quantum conformal field theory, Comm. Math. Phys. 123 (1989), $177-254$.

[103] Morozov A., String theory: what is it?, Phys. Usp. 35 (1992), 671-714.

[104] Morozov A., Integrability and matrix models, Phys. Usp. 37 (1994), 1-55, hep-th/9303139.

[105] Morozov A., Challenges of matrix models, hep-th/0502010.

[106] Morozov A., Matrix models as integrable systems, hep-th/9502091.

[107] Morozov A., Zenkevich Y., Decomposing Nekrasov decomposition, J. High Energy Phys. 2016 (2016), no. 2, 098, 44 pages, arXiv:1510.01896.

[108] Nekrasov N., Seiberg-Witten prepotential from instanton counting, Adv. Theor. Math. Phys. 7 (2003), 831-864, hep-th/0206161.

[109] Nekrasov N., Okounkov A., Seiberg-Witten theory and random partitions, hep-th/0306238.

[110] Nekrasov N., Shatashvili S., Quantization of integrable systems and four dimensional gauge theories, in XVIth International Congress on Mathematical Physics, World Sci. Publ., Hackensack, NJ, 2010, 265-289, arXiv:0908.4052.

[111] Nemkov N., S-duality as Fourier transform for arbitrary $\epsilon_{1}, \epsilon_{2}$, J. Phys. A: Math. Theor. 47 (2014), 105401, 15 pages, arXiv:1307.0773.

[112] Nemkov N., On modular transformations of toric conformal blocks, J. High Energy Phys. 2015 (2015), no. 10, 037, 26 pages, arXiv:1504.04360.

[113] Nemkov N., Fusion transformations in Liouville theory, Theoret. and Math. Phys. 189 (2016), 1574-1591, arXiv:1409.3537.

[114] Novikov S.P., A method for solving the periodic problem for the KdV equation and its generalizations, Rocky Mountain J. Math. 8 (1978), 83-93.

[115] Okounkov A., Reshetikhin N., Vafa C., Quantum Calabi-Yau and classical crystals, in The Unity of Mathematics, Progr. Math., Vol. 244, Birkhäuser Boston, Boston, MA, 2006, 597-618, hep-th/0309208.

[116] Orantin N., Symplectic invariants, Virasoro constraints and Givental decomposition, arXiv:0808.0635.

[117] Ponsot B., Teschner J., Liouville bootstrap via harmonic analysis on a noncompact quantum group, hep-th/9911110.

[118] Ponsot B., Teschner J., Clebsch-Gordan and Racah-Wigner coefficients for a continuous series of representations of $\mathcal{U}_{q}(\mathfrak{s l}(2, \mathbb{R}))$, Comm. Math. Phys. 224 (2001), 613-655, math.QA/0007097.

[119] Shintani T., On a Kronecker limit formula for real quadratic fields, J. Fac. Sci. Univ. Tokyo Sect. IA Math. 24 (1977), 167-199.

[120] Teschner J., From Liouville theory to the quantum geometry of Riemann surfaces, hep-th/0308031.

[121] Witten E., On the structure of the topological phase of two-dimensional gravity, Nuclear Phys. B 340 (1990), 281-332.

[122] Wyllard N., $A_{N-1}$ conformal Toda field theory correlation functions from conformal $\mathcal{N}=2 \mathrm{SU}(N)$ quiver gauge theories, J. High Energy Phys. 2009 (2009), no. 11, 002, 22 pages, arXiv:0907.2189.

[123] Zamolodchikov A.B., Zamolodchikov A.B., Conformal field theory and critical phenomena in twodimensional systems, Soviet Sci. Rev. A Phys. 10 (1989), 269-433. 